\title{
Why Aren't There More Maternal Deaths? A Decomposition Analysis
}

\author{
John A. Ross • Ann K. Blanc
}

Published online: 24 March 2011

(C) The Author(s) 2011. This article is published with open access at Springerlink.com

\begin{abstract}
Globally, the number of maternal deaths remains large, and the risk per birth is high in the developing world. Deaths declined between 1990 and 2008, despite the $42 \%$ increase in women. We decompose selected determinants to help explain the decline. Numbers of women, births, and fertility rates come from the UN; maternal mortality ratios are from the UN and from Hogan et al. Decomposition isolates the effects of additional women, decreases in fertility, and declines in mortality ratios, also in rates. Women aged 15-49 increased by $42 \%$, but births remained constant due to declining fertility rates. The fertility decline alone averted approximately 1.7 million deaths, 1990-2008. The risk per birth (MMR) also fell, adding to the decline in the number of deaths. Exceptional declines occurred in the maternal mortality rate. SubSaharan Africa has experienced minimal declines in deaths, due to increases in women and small declines in fertility and mortality. The growing numbers of women have made international efforts to reduce the number of maternal deaths ever more challenging. Comparatively little attention has been given to the offsetting effect of the historic fertility declines in the developing world, and hence a flat trend in births. The maternal mortality ratio has also fallen, reflecting the success of direct maternal health efforts. Programs that provide couples with the means to control their fertility can reinforce fertility declines. These
\end{abstract}

J. A. Ross

Futures Group, One Thomas Circle, NW,

Washington, DC 20005, USA

e-mail: rosshome8@frontiernet.net

A. K. Blanc $(\bowtie)$

Maternal Health Task Force, EngenderHealth,

440 Ninth Avenue, New York, NY 10001, USA

e-mail: ablanc@engenderhealth.org programs are companions to ongoing, direct measures to reduce the risk of death once pregnant.

Keywords Maternal mortality $\cdot$ Fertility $\cdot$ Maternal death rates - Sub-Saharan Africa - South Asia - Decomposition

\section{Background/Significance}

The fifth goal of the eight Millennium Development Goals adopted by the member states of the United Nations in 2000 aims to improve maternal health, with a specific target to reduce the maternal mortality ratio to $75 \%$ of its 1990 level by 2015 . A recent report by the Secretary General concludes that, at the current rate of progress, this target "cannot be achieved" [1] and that substantially accelerated progress is needed. After several decades of debate, the maternal health field has reached a consensus on the most effective means by which to reduce maternal mortality [2]. This consensus focuses on four core strategies: family planning with related reproductive health services (including safe abortion where legal), skilled care during pregnancy and childbirth, emergency obstetric care, and immediate postnatal care. While all four strategies contribute to reducing the number of maternal deaths, ${ }^{1}$ the latter three do so by reducing risks among women who are (or recently were) pregnant. In contrast, family planning reduces the number of deaths in two ways: first, by reducing fertility (i.e., the number of women who become pregnant) and second, by reducing the risk associated with each birth through shifting the

\footnotetext{
1 The World Health Organization defines a maternal death as the death of a woman while pregnant or within 42 days of termination of pregnancy, irrespective of the duration and site of the pregnancy, from any cause related to or aggravated by the pregnancy or its management but not from accidental or incidental causes.
} 
composition of births away from older mothers and higher parities, among which the risk is comparatively high. ${ }^{2}$ Time trends within 36 developing countries show the common movement through time between a declining proportion of births that carry high mortality risks and a rising proportion of women using contraception, which especially reduces high-parity births [3].

While the effect of family planning on maternal deaths has received some attention in the family planning literature [4-7], it has been much less prominent in the maternal health literature, which has tended to emphasize the reduction of risk among pregnant women ${ }^{3}$ [8]. However, some recent formulations of policy strategies for improving maternal health have explicitly recognized the importance of contraceptive use $[9,10]$.

The objective of this article is to assess the relative contributions of declines in fertility and in the risk of maternal death on the number of maternal deaths. We decompose trends in the number of maternal deaths between 1990 and 2008 into three components: the number of women of reproductive age, the general fertility rate, and the maternal mortality ratio. We also examine trends in the maternal mortality rate (not the ratio), which has been virtually ignored in the international discourse on maternal health. We present results for the developing world as a whole, as well as for the two regions that account for most of the maternal deaths that occur annually: sub-Saharan Africa and South Asia. Among the countries in these regions, India has the most deaths by a large margin, 62,000 in 2008 , followed by Nigeria at 50,000 . Other countries in these regions fall below those numbers, for example the D.R. Congo at 19,000, Bangladesh at 18,000, then Pakistan, Ethiopia, and Tanzania at 14,000 each.

\section{Data and Methodology}

The number of maternal deaths is calculated from three components: the number of women of reproductive age, 15-49 (WRA); the general fertility rate (GFR): births per year per 1,000 women of reproductive age; and the

\footnotetext{
${ }^{2}$ Family planning use may also reduce maternal mortality through lengthening the duration of intervals between births although there is little evidence on this point.

${ }^{3}$ Previous studies attempting to quantify the effect of contraceptive use on maternal mortality, published mostly in the 1980s, were hampered by a lack of data. At the time, many countries had no reliable estimates of maternal mortality at all and time series data were virtually non-existent except in a few cases. While maternal mortality data are still inadequate and are often unreliable, considerable effort has been put into data improvements over the last 15 years-through the inclusion of the topic in population surveys, censuses, and other data collection methods-and global and regional estimates have now been produced for several time periods.
}

maternal mortality ratio (MMR): the number of maternal deaths per 100,000 live births. The first two, multiplied together, generate the number of births, which is then multiplied by the MMR to give the number of maternal deaths. This information is estimated for every country in the developing world. The population data are available from the UN Population Division's 2008 series [11], and the MMR estimates are available from the set recently released by collaborating UN agencies [12]. This UN series updates and replaces estimates issued by the same agencies for 1990, 1995, 2000, and 2005 [13]. However the latest release for 2008 radically revises the earlier work, finding for the first time a sharp downward trend in the MMR based upon an augmented data set and improved methods. Some reassurance as to the validity of the revision comes from rather close agreement between it and another recent set of MMR estimates by Hogan et al. [14], although the two sets of estimates differ in the speed of MMR decline after 1990 (Fig. 4).

For the developing world as a whole, and for the two major regions that account for most deaths, we follow the 1990-2008 trends to obtain the relative change in each of the three determinants. The purpose is to quantify the role of the three components in determining the number of deaths. Since the number of maternal deaths is simply the product of the number of women, the GFR, and the MMR, the effect of each can be obtained by holding the 1990 value constant to 2008 and noting the difference from the actual trend. That, together with the ratios of change, provides a picture of the role of each of the three determinants.

Second, we decompose changes in the maternal death rate, the number of deaths per 1,000 women aged 15-49 per year. Note that while the denominator of the MMR is the number of births, the denominator of the maternal death rate is the number of women. Thus, the MMR measures the risk of death at each birth whereas the maternal death rate measures the average woman's annual probability of death from maternal causes. That analysis parallels the one above: changes in fertility and in the MMR are used along with changes in the numbers of women to gauge the effect of each component.

"Fertility decline" here is the general fertility rate, which historically has responded primarily to increases in contraceptive use, including sterilization. Over the 1990-2008 period the postponement of marriage has also reduced fertility in some countries. Further, increases in abortion, or decreases in breastfeeding, may have had effects on fertility that have not been well measured.

\section{Results}

As shown in Fig. 1, the number of women of reproductive age in the developing world increased by $42 \%$ between 


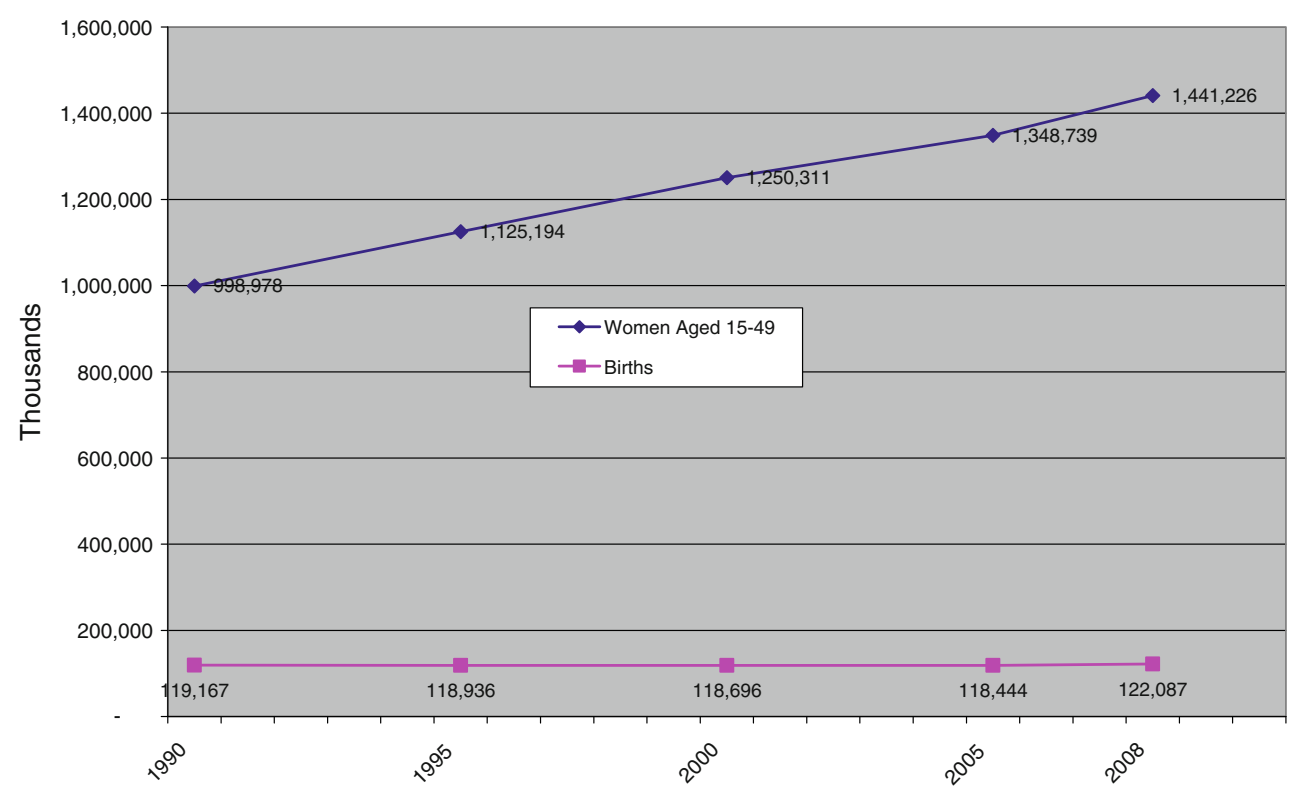

Fig. 1 Numbers of women aged 15-49 and births, developing countries, 1990-2008

1990 and 2008, from about one billion to 1.4 billion. The growing numbers of women have made international efforts to reduce the number of maternal deaths ever more challenging. In the other direction however, fertility fell enough from 1990 to 2008 to offset that growth. Therefore, the number of births has stayed essentially flat, which has averted large increases in burdens on health services over the years.

In Table 1 the annual number of maternal deaths in 1990 $(541,000)$ declined to 355,000 in 2008 . The number of deaths is simply the product of the three determinants, so that a percentage change in any one produces the same percentage change in deaths. For all developing countries, the rise of $42 \%$ in the number of women has been counterbalanced by the decline in fertility and by the decline in the MMR, together resulting in a $34 \%$ decline in annual deaths over the 18 year period.

The picture is quite different for sub-Saharan Africa, where the number of women rose by a full two-thirds. Working against that trend was an $18 \%$ fall in the GFR, holding the rise in births to $37 \%$. The one-fourth fall (26\%) in the MMR, acting on births, helped keep the number of 2008 deaths close to the number in 1990, though with intermediate increases.

The South Asia experience contrasts sharply with that of sub-Saharan Africa. Although the number of women rose by half $(52 \%)$ the GFR fell by a full third (33\%); consequently the number of births was flat. The MMR decline was especially notable at well over half, and deaths fell equally.

The effects of the three determinants are decomposed in Table 2 and Fig. 2. By simply holding the 1990 figure for the GFR constant, for example, the number of deaths in each 5-year period increases. The difference between those results and the actual results gives the estimate of the contribution of the fertility decline to the number of deaths over time. Similar calculations show the effect of the WRA increase as an upward force on deaths, for a cumulative difference of 1,252,000 deaths between the actual and the simulated calculation. That is, with all else equal, if the number of women had not increased from 1990 to 2008, over 1.2 million deaths would have been averted, including 851,000 in sub-Saharan Africa and 514,000 in South Asia. There is a downward force from the GFR decline of 1,672,000 deaths, and a downward force from the MMR decline of 1,492,000 deaths. In addressing the tragedy of maternal deaths in the developing world, the focus has been primarily upon the risk per pregnancy/birth (the MMR), but if the fertility rate had remained high and constant, the total number of maternal deaths over the 18 years between 1990 and 2008 would have been about 1.7 million higher, an increase of a fifth $(20 \%)$, over the actual number of 8.2 million.

In sub-Saharan Africa and South Asia the upward effect of the WRA increases amounted to additions of 851,000 deaths and 514,000 deaths, respectively. In South Asia, the effect of the MMR decline was about double that of the GFR; the relative contribution of fertility decline was $35 \%$ compared to $65 \%$ for the MMR decline. That ratio was nearly even in sub-Saharan Africa at $49-51 \%$ for the GFR and MMR effects, respectively. For developing countries as a whole it was $53-47 \%$, giving about equal weight to the fertility decline and the MMR decline in the overall reduction of maternal deaths. 
Table 1 Maternal deaths and death rates: effects of three determinants

\begin{tabular}{|c|c|c|c|c|c|c|}
\hline & $\begin{array}{l}\text { 1. Women aged } \\
15-49(000 \mathrm{~s})\end{array}$ & 2. GFR* & 3. Births (000s) & 4. $\mathrm{MMR}^{* *}$ & $\begin{array}{l}\text { 5. Annual number } \\
\text { of maternal deaths }\end{array}$ & $\begin{array}{l}\text { 6. Maternal death } \\
\text { rates**** }\end{array}$ \\
\hline \multicolumn{7}{|c|}{ Developing countries } \\
\hline 1990 & $1,018,523$ & 119 & 121,623 & 445 & 540,622 & 0.53 \\
\hline 1995 & $1,137,370$ & 106 & 120,347 & 411 & 494,254 & 0.43 \\
\hline 2000 & $1,260,612$ & 95 & 119,782 & 374 & 448,569 & 0.36 \\
\hline 2005 & $1,376,677$ & 88 & 120,988 & 323 & 390,699 & 0.28 \\
\hline 2008 & $1,441,226$ & 85 & 122,087 & 291 & 355,238 & 0.25 \\
\hline Ratios 2008/1990 & 1.42 & 0.71 & 1.00 & 0.65 & 0.66 & 0.46 \\
\hline \multicolumn{7}{|l|}{ Sub-Saharan Africa } \\
\hline 1990 & 117,149 & 196 & 22,928 & 867 & 198,799 & 1.70 \\
\hline 1995 & 136,205 & 185 & 25,229 & 858 & 216,493 & 1.59 \\
\hline 2000 & 157,197 & 176 & 27,629 & 795 & 219,577 & 1.40 \\
\hline 2005 & 179,924 & 168 & 30,160 & 710 & 214,171 & 1.19 \\
\hline 2008 & 194,797 & 161 & 31,447 & 646 & 203,086 & 1.04 \\
\hline Ratios 2008/1990 & 1.66 & 0.82 & 1.37 & 0.74 & 1.02 & 0.61 \\
\hline \multicolumn{7}{|l|}{ South Asia } \\
\hline 1990 & 267,261 & 141 & 37,643 & 615 & 231,532 & 0.87 \\
\hline 1995 & 303,825 & 126 & 38,394 & 512 & 196,631 & 0.65 \\
\hline 2000 & 343,916 & 112 & 38,518 & 434 & 167,020 & 0.49 \\
\hline 2005 & 382,702 & 100 & 38,307 & 333 & 127,598 & 0.33 \\
\hline 2008 & 406,120 & 94 & 38,083 & 285 & 108,386 & 0.27 \\
\hline Ratios 2008/1990 & 1.52 & 0.67 & 1.01 & 0.46 & 0.47 & 0.31 \\
\hline
\end{tabular}

col. 3 = product of col. 1 and col. 2 , Col. $5=$ product of col. 3 and col. 4 , Col. $6=$ col. 5 divided by col. 1

* GFR general fertility rate (births/year/1000 women aged 15-49)

** MMR maternal deaths $/ 100,000$ births

*** Maternal death rate: deaths/year/1,000 women aged 15-49

Action programs cannot change the current numbers of childbearing age women, but they can affect both fertility and mortality trends, and the two interact. For example, national family planning and reproductive health programs have helped to reduce the number of births, while specific health interventions have helped to reduce the risk per birth, and the two have been synergistic. Their interactive effect is estimated in Table 2, last column, by keeping constant the initial GFR and MMR values during the whole period. Had there been no changes the high MMR would have been acting on far more births; consequently, $3,625,000$ more deaths would have occurred, more than the sum of 1,672,000 and 1,492,000 added deaths from each component acting alone.

\section{Maternal Mortality Rates, not Ratios}

Little attention has been devoted to the maternal death rate, i.e., the number of maternal deaths per year per thousand women aged 15-49. Low maternal mortality rates can occur in the presence of high MMRs if the fertility rate is low and few women are exposed to the risks of pregnancy.
It is therefore of interest to decompose the change in maternal mortality rates into the portions due to the fertility decline and to the MMR decline. The maternal mortality rates in Table 1 (last column) seem low: in 2008 there were only 0.25 deaths per thousand women overall and only one death per thousand women in sub-Saharan Africa. The rates are relatively low because many women do not give birth in any given year, and even the limited numbers of births are subjected to an average mortality rate below a third of a percent (at an MMR of 291 in 2008). Thus, among 1,000 women there may be 85 births in a year (in 2008), which multiplied by $0.291 \%$ yields only 0.25 deaths per thousand women.

The rates have fallen substantially due to the great increase in the denominator of women over the 18 years, and by the constraint on deaths in the numerator due to the GFR and MMR declines. Between 1990 and 2008, for all developing countries, the maternal mortality rate fell by $54 \%$ (Fig. 3), and the literature in this field has overlooked this advance. Equally significant however, is that both the ratio and the rate for the developing world remain far above those in developed countries. 
Table 2 Maternal deaths: gains and losses from three determinants

\begin{tabular}{|c|c|c|c|c|c|}
\hline & $\begin{array}{l}\text { Actual maternal deaths } \\
\text { during } 5 \text {-year periods }\end{array}$ & $\begin{array}{l}\text { Maternal deaths if no } \\
\text { WRA* increase }\end{array}$ & $\begin{array}{l}\text { Maternal deaths if no } \\
\text { GFR** decline }\end{array}$ & $\begin{array}{l}\text { Deaths if no } \\
\text { MMR*** decline }\end{array}$ & $\begin{array}{l}\text { Deaths with both GFR } \\
\& \text { MMR constant }\end{array}$ \\
\hline \multicolumn{6}{|l|}{ Developing countries } \\
\hline 1990-1995 & $2,587,191$ & $2,458,075$ & $2,745,997$ & $2,688,936$ & $2,860,819$ \\
\hline $1995-2000$ & $2,357,058$ & $2,012,583$ & $2,803,735$ & $2,668,485$ & $3,182,065$ \\
\hline $2000-2005$ & $2,098,170$ & $1,628,702$ & $2,736,434$ & $2,675,605$ & $3,499,620$ \\
\hline $2005-2008$ & $1,118,905$ & 810,156 & $1,547,421$ & $1,620,725$ & $2,243,574$ \\
\hline Total & $8,161,324$ & $6,909,517$ & $9,833,587$ & $9,653,752$ & $11,786,079$ \\
\hline Excess deaths $* * * * *$ & & $-1,251,807$ & $1,672,263$ & $1,492,428$ & $3,624,755$ \\
\hline \multicolumn{6}{|l|}{ Sub-Saharan Africa } \\
\hline 1990-1995 & $1,038,229$ & 962,505 & $1,068,896$ & $1,043,854$ & $1,074,840$ \\
\hline $1995-2000$ & $1,090,174$ & 874,598 & $1,183,186$ & $1,145,741$ & $1,244,744$ \\
\hline $2000-2005$ & $1,084,370$ & 757,708 & $1,236,445$ & $1,252,640$ & $1,430,218$ \\
\hline $2005-2008$ & 625,886 & 392,371 & 744,420 & 801,246 & 953,841 \\
\hline Total & $3,838,659$ & $2,987,182$ & $4,232,947$ & $4,243,481$ & $4,703,643$ \\
\hline Excess deaths $* * * * *$ & & $-851,476$ & 394,288 & 404,822 & 864,984 \\
\hline \multicolumn{6}{|l|}{ South Asia } \\
\hline 1990-1995 & $1,070,409$ & $1,011,251$ & $1,126,730$ & $1,169,209$ & $1,236,850$ \\
\hline $1995-2000$ & 909,128 & 756,903 & $1,072,998$ & $1,182,669$ & $1,402,868$ \\
\hline $2000-2005$ & 736,543 & 547,253 & 973,963 & $1,181,329$ & $1,573,700$ \\
\hline 2005-2008 & 353,976 & 240,653 & 513,509 & 704,788 & $1,025,052$ \\
\hline Total & $3,070,056$ & $2,556,060$ & $3,687,201$ & $4,237,995$ & $5,238,471$ \\
\hline Excess deaths $* * * * *$ & & $-513,996$ & 617,145 & $1,167,939$ & $2,168,415$ \\
\hline
\end{tabular}

* WRA women of reproductive age (15-49)

** GFR General fertility rate (births/year/1,000 women aged 15-49)

*** MMR: maternal deaths $/ 100,000$ births

**** Deaths in five years $=5$ times the mean of deaths in starting and ending years from Table 1

$* * * * *$ Added deaths $=$ total in each col. minus total of col. 1

For sub-Saharan Africa, a lesser decline in the rate of $39 \%$ occurred, from the high level of 1.70-1.04. Even though the number of deaths was basically flat, the number of women increased by two-thirds. The South Asia picture is again more favorable. There the maternal mortality rate fell by $69 \%$, from the lower starting level of $0.87-0.27$. That was due to the double effect of an appreciable fall of $54 \%$ in the estimated MMR and a 33\% fall in the fertility rate.

These declines in maternal death rates outpace the declines in numbers of deaths, which remain under pressure from the large increases in numbers of women. In contrast, the rates are on a per-woman basis and reflect just the two determinants of fertility and MMR risk.

\section{An Alternative Estimate of Maternal Mortality Ratios}

During 2010, the global picture of maternal mortality was considerably changed by two publications. The first to appear, by Hogan et al. [14] estimated the world-wide
MMR for 2008 at only 251, and the number of deaths at only 343,000 . Both were far below the accepted figures, which came from the series issued by cooperating UN agencies that had produced estimates every 5 years starting in 1990, through 2005. The new estimates released by these agencies in 2010 however, are lower and relatively consistent with the Hogan et al. series. While Hogan et al. note in passing the effect of declining fertility on the MMR, they do not attempt to quantitatively estimate its impact.

We have calculated alternative results with the Hogan et al. MMR values, noting however, that they do not affect the savings in the absolute numbers of deaths due to the historic fertility declines, which have held the number of births essentially constant. The MMR reductions are quite separate, and different MMR schedules do not affect the fertility decline component. They concern only the mortality risk once pregnant.

Some of the Hogan et al. results are close to those from the UN-based series, e.g. 343,000 and 355,000 deaths, respectively for 2008 . However the two trend patterns after 


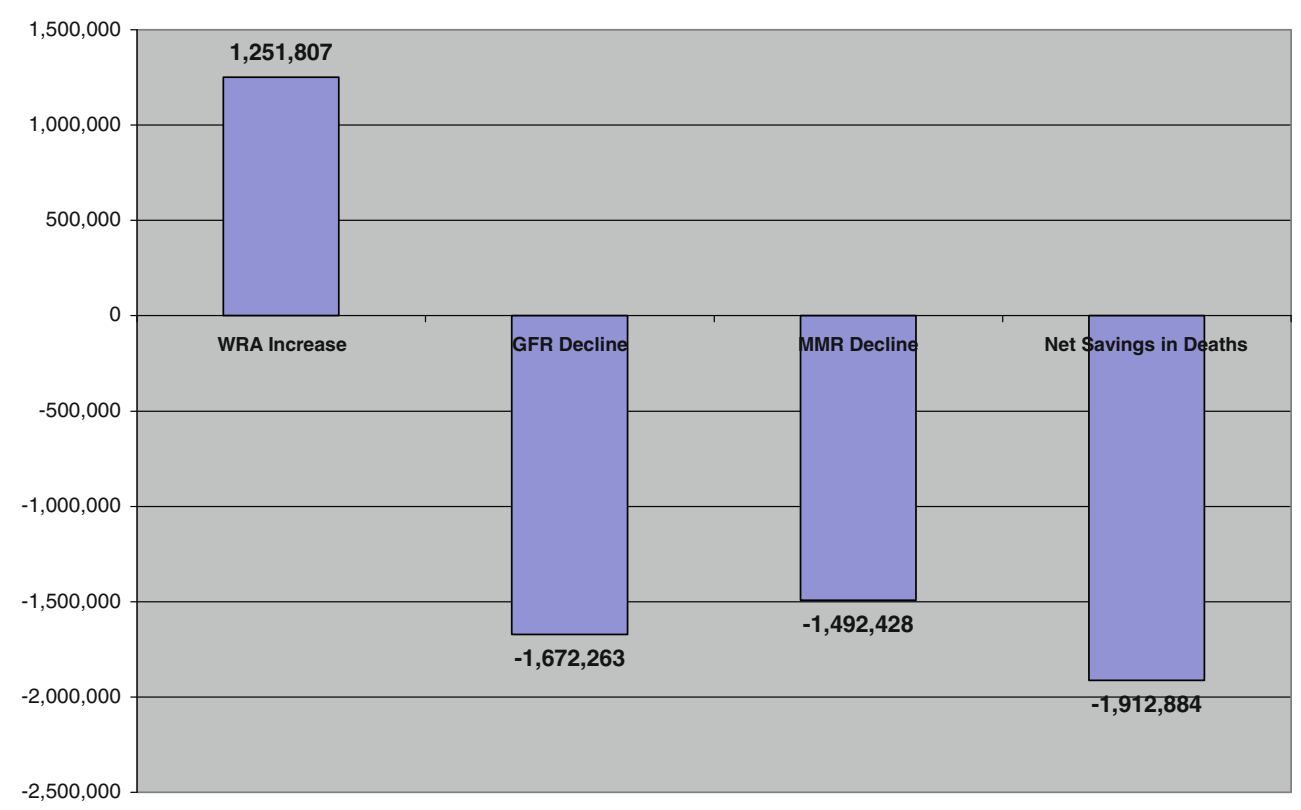

Fig. 2 Effects on numbers of deaths, developing countries, 1990-2008

Fig. 3 Declines in the maternal mortality rate (annual deaths per 1,000 women)

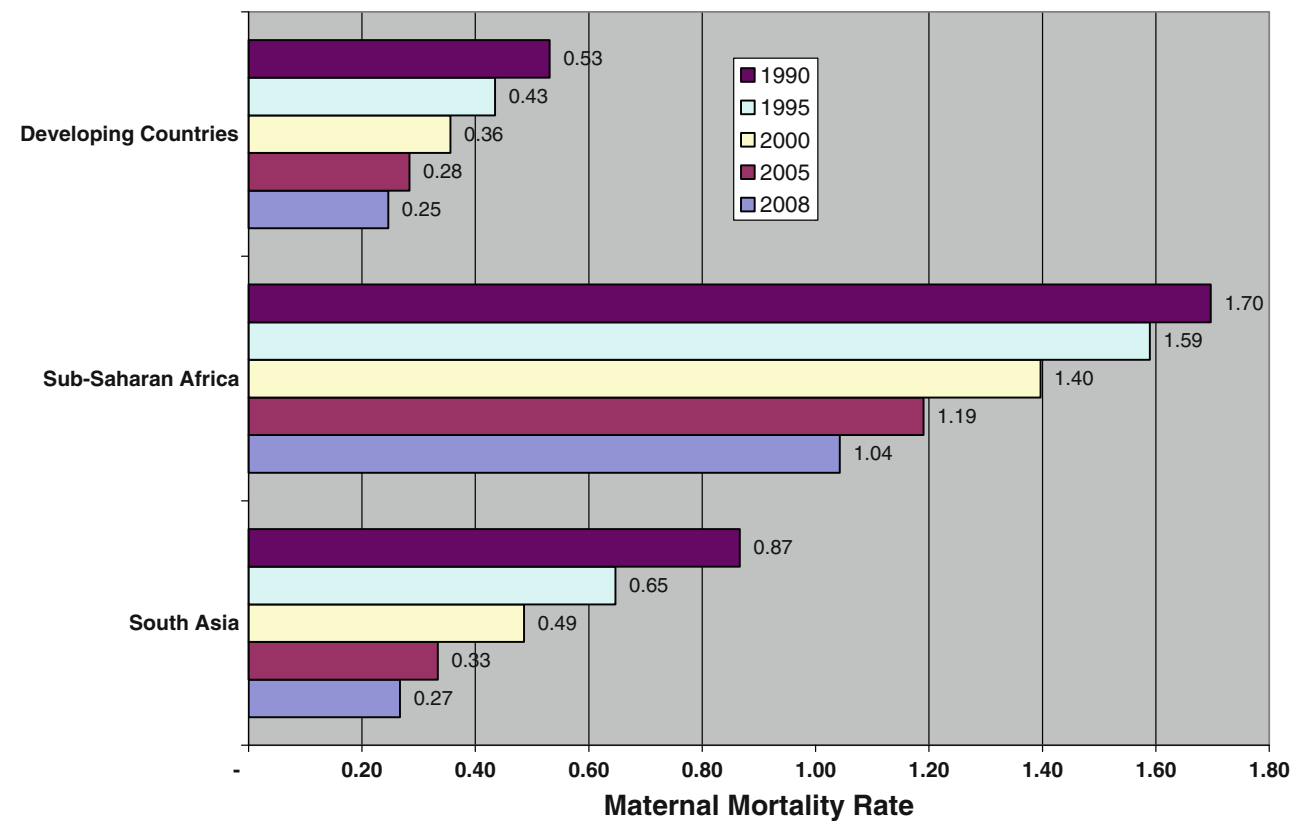

1990 are dissimilar (Fig. 4 top lines), with the UN-based set starting higher and declining more evenly. The agreement is close for South Asia in both level and pattern, but less so for sub-Saharan Africa. Differences between the two sets of estimates arise from several sources; as summarized in the UN report they include somewhat different types of data sources and adjustments to them, as well as different modeling strategies, mortality schedules, and treatments of HIV/AIDS-related maternal deaths.

The maternal death rates are also close; for example in 2008 the two sources found, for the developing world, rates of 0.25 and 0.24 by the UN and by Hogan et al., respectively. For sub-Saharan Africa the two estimates were 1.04 and 0.90; for South Asia they were 0.27 and 0.30, respectively.

\section{Discussion}

The decomposition approach to changes over time works well when there are clearly defined determinants and longterm information is available, subject to measurement 
Fig. 4 Numbers of maternal deaths by area and source

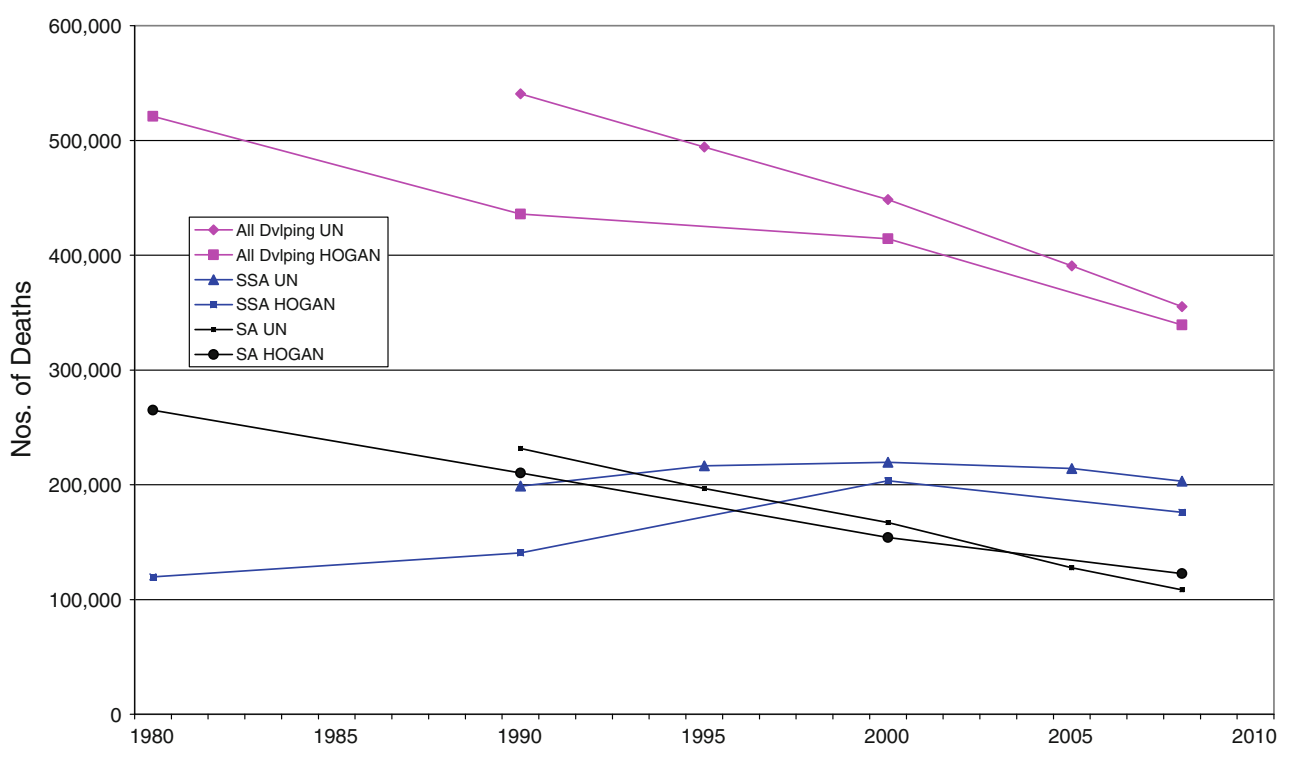

errors in the inputs. Strengths of the approach include the precision of the equations and in this case, applications to the developing world as a whole and to the two regions that experience most of the maternal deaths. Estimates are possible for the effect of each determinant separately and for interactive effects between them. The limitations of any application include the well known and substantial empirical errors in estimates of maternal deaths, as well as those to which numbers of women and births are subject. One definitional reservation pertains to the MMR, which is based on births, whereas many maternal deaths occur during pregnancy. Using pregnancies (for which no internationally comparable data exist) would enlarge the denominator and have the effect of reducing the ratio, but would not change the counts of absolute numbers of deaths in the numerator.

The average woman's annual risk of a maternal death, the maternal death rate, fell by over half in the 18-year period examined here. Without the past decline in fertility of nearly one-third, and the decline in the MMR of over a third, the numbers of maternal deaths would have been about $44 \%$ higher, with 3.6 million more deaths between 1990 and 2008.

The decline in annual deaths from 541,000 in 1990-355,000 in 2008 has been achieved despite the growth in numbers of women. The particular contribution of the fertility decline has been to keep the absolute number of births constant; that has been overlooked in the literature of the field, but the effect is of the same magnitude as that of the decline in the MMR. There has also been an interaction between the two effects, first by averting many births on which the MMR would otherwise have acted, and second because greater contraceptive use has also helped lower the average MMR by improving the mix of births according to their risk profiles, shifting births away from high parities and older ages of mothers [3].

The overall decline in deaths is an advance, but it is region-specific. South Asia has experienced substantial reductions in both fertility and in the MMR risk, cutting annual deaths by more than half. In sub-Saharan Africa on the other hand the total number of deaths is the same now as in 1990, and this region contains some of the largest and least developed countries. Fertility rates have fallen there, but less than in any other region. Other research shows the relatively high desired family sizes there and the weakness of the national family planning programs in many countries [14]; moreover the region suffers from low educational attainment, a continuing HIV epidemic, and high poverty levels [15].

In a context of scarce resources, a strategic consideration for reducing deaths further concerns the relative emphasis on contraceptive increase and on direct interventions to lower the risks during pregnancy. Given the equal share of credit for total savings in deaths for the fertility decline and the MMR decline, and in light of the interactions demonstrated above, it is clear that both are needed and each will reinforce the other. A sharper focus is needed in most developing countries to both prevent unplanned pregnancies and to lower the risks during pregnancy. Programs that satisfy unmet needs for contraception are strong allies to programs that improve survival during pregnancy and birth.

Both types of efforts face the common challenge of upgrading the quality of health systems that are basic to family planning and maternal health programs, to extend services into urban slums and outward to rural populations. The priority needs are to expand and sustain access to multiple contraceptive methods, post abortion care, 
emergency obstetric services, and skilled care at delivery and afterward.

Acknowledgments This research was supported by the Maternal Health Task Force at EngenderHealth through a grant from the Bill \& Melinda Gates Foundation. This article is based upon a paper presented at the International Conference on Family Planning: Research and Best Practices, Kampala, Uganda, November 15-18, 2009. JAR conceived the study, carried out the statistical analysis, and contributed to interpretation of the data, drafting and editing of the paper. $\mathrm{AKB}$ contributed to interpretation of the data, drafting, and editing of the paper. We declare that we have no conflicts of interest.

Open Access This article is distributed under the terms of the Creative Commons Attribution Noncommercial License which permits any noncommercial use, distribution, and reproduction in any medium, provided the original author(s) and source are credited.

\section{References}

1. United Nations. (2010). Keeping the promise: A forward-looking review to promote an agreed action agenda to achieve the Millennium Development Goals by 2015.Report of the Secretary General. New York: United Nations.

2. Deliver, Women. (2010). Focus on five: Women's health and the MDGs. New York: Women Deliver.

3. Stover, J., \& Ross, J. (2010). How increased contraceptive use has reduced maternal mortality. Maternal and Child Health Journal, 14(5), 687-695.

4. Fortney, J. A. (1987). The importance of family planning in reducing maternal mortality. Studies in Family Planning, 18(2), 109-114.
5. Winikoff, B., \& Sullivan, M. (1987). Assessing the role of family planning in reducing maternal mortality. Studies in Family Planning, 18(2), 128-143.

6. Trussell, J., \& Pebley, A. (1984). The potential impact of changes in fertility on infant, child, and maternal mortality. Studies in Family Planning, 15, 267-280.

7. Blacker, J. G. C. (1987). Health impacts of family planning. Health Policy and Planning., 2(3), 193-203.

8. Campbell, O. M. R., \& Graham, W. (2006). Strategies for reducing maternal mortality: Getting on with what works. The Lancet, 368(9543), 1284-1299.

9. http://www.who.int/pmnch/events/2009/20090923_mnchconsen susstory/en/index2.html. Accessed 10/16/2009.

10. Ki-Moon, B. (2010). Global strategy for women's and children's health. New York: United Nations.

11. United Nations Population Division. (2008). World population prospects: The 2008 revision, Vol. I: Comprehensive tables. New York: United Nations.

12. WHO, UNICEF, UNFPA, \& World Bank. (2010). Trends in maternal mortality 1990 to 2008. Geneva: WHO.

13. WHO, UNICEF, UNFPA, \& World Bank. (2007). Maternal mortality in 2005: Estimates developed by WHO, UNICEF, UNFPA, and The World Bank. Geneva: WHO.

14. Hogan, M.C., Foreman, K.J., Naghavi, M., Ann, S.Y., Wang, M., Makela, S.M., Lopez, A.D. Lozano, R., Murray. C. (2010). Maternal mortality for 181 countries, 1980-2008: a systematic analysis of progress towards Millennium Development Goal 5. Published on line April 22, 2010. The Lancet, pp. 1-5. doi: 10.1016/SO140-6736(10)60518-1.

15. Ross, J., \& Smith, E. (2010). The family planning effort index: 1999, 2004, and 2009. Washington DC: Futures Group, Health Policy Initiative. Published on line. 